\title{
Without Track Changes
}

1 Running head: Surgical navigation in canine total knee replacement

2

3 Full Title: Surgical Navigation Improves the Precision and Accuracy of Tibial

4 Component Alignment in Canine Total Knee Replacement

6 Authors: Kaleigh M. Peters MS; Erin Hutter PhD; Robert A. Siston PhD; Judith Bertran

7 Ldo Med Vet; Matthew J. Allen, Vet MB, PhD

9 Institutional Affiliations: Departments of Veterinary Clinical Sciences and Mechanical 10 \& Aerospace Engineering, The Ohio State University, Columbus, $\mathrm{OH}$.

11 Work performed at: The Ohio State University, Columbus, $\mathrm{OH}$.

12 Financial support: This work was supported in part through the Summer Research 13 Program at the College of Veterinary Medicine, The Ohio State University, Columbus, OH.

15 Prior presentation: Presented in part at the 4th World Veterinary Orthopedic 16 Congress, Breckenridge, CO (April 1-4, 2014).

\section{Correspondence should be addressed to:}

19 Matthew J. Allen, Vet MB, PhD

20 Department of Veterinary Medicine

21 University of Cambridge

22 Madingley Road

23 Cambridge CB3 0ES

24 United Kingdom

25 Tel: +44 1223337642

26 Fax: +44 1223337610

27 E-mail: mja1000@cam.ac.uk

29 Conflict of interest: One of the authors (MJA) is a consultant for the BioMedtrix total 30 knee replacement program. 


\section{ABSTRACT}

34 Objective -- The goal of the current study was to determine whether computer-assisted 35 surgical navigation improves the accuracy of tibial component alignment in canine TKR.

36 Study design - Retrospective radiographic review and prospective ex-vivo study.

37 Sample population -- Seventeen sets of canine total knee replacement (TKR) 38 radiographs were reviewed to determine the incidence and magnitude of tibial 39 component

40 malalignment. A cadaveric study was then performed in 12 canine stifle joints.

41 Methods -- Tibial component alignment was compared after either standard ("surgeon42 guided") component placement or computer-assisted ("navigation-guided") placement. 43 Results were compared against the current recommendations of a neutral $\left(0^{\circ}\right.$ varus44 valgus) ostectomy in the frontal plane and $6^{\circ}$ of caudal slope in the sagittal plane.

45 Results - Malalignment of greater than $3^{\circ}$ in the frontal and sagittal planes was 46 identified in $12 \%$ and $24 \%$ of radiographs respectively. Surgical navigation reduced 47 both the mean error $(\mathrm{p}=0.007)$ and the variability in frontal plane alignment $(\mathrm{p}<0.001)$ 48 as compared with surgeon-guided procedures. The mean error in sagittal plane 49 alignment was not significantly different $(\mathrm{p}=0.321)$ but variability in alignment was 50 significantly lower when navigation was used $(\mathrm{p}=0.008)$.

51 Conclusions -- Surgical navigation significantly improves accuracy and decreases 52 variability in tibial component alignment in canine TKR. Clinical trials would be required 53 to determine whether these improvements in surgical accuracy lead to better clinical 54 outcomes in terms of joint function and a reduction in long-term implant wear. 


\section{INTRODUCTION}

57 Until relatively recently, treatment options for end-stage canine stifle disease have been

58 limited to medical management, arthrodesis, or amputation. The presence of bilateral

59 disease often makes these options less feasible due to contralateral stifle pathology.

60 Total knee replacement (TKR) is now an established treatment option for dogs with

61 severe osteoarthritis secondary to multiple primary pathologies [1]. The primary goals

62 of TKR are to relieve pain and to improve stifle joint function. Approximately 350 canine

63 TKR procedures have been performed around the world to date (personal 64 communication, BioMedtrix LLC).

66 The long-term outcome of TKR depends on careful preparation of the articular surfaces. 67 The accuracy of conventional ("surgeon-guided") operative technique is largely 68 dependent on three factors: experience of the surgeon, quality of instrumentation, and 69 the inherent anatomy of the patient. In humans, it has been suggested that the most 70 common cause of revision TKR is surgical error [2]. An estimated 10\% of TKR 71 procedures result in errors in tibial and femoral alignment of $>3^{\circ}$ even when performed 72 by highly experienced surgeons [2]. Errors in tibial component positioning are 73 associated with an increased risk of implant wear, mechanical loosening and pain [3,4]. 74 In an attempt to improve surgical accuracy, some surgeons have turned to the use of 75 computer-assisted surgical navigation systems that use three-dimensional motion 76 capture and optical tracking technologies to collect real-time data on bone and joint 77 alignment in the operating room.

79 Surgical navigations systems can be broadly classified into image-based systems and 80 image-free systems. With image-based systems, pre-operative imaging (typically MRI or $81 \mathrm{CT}$ ) or intra-operative imaging (fluoroscopy) are used to generate a three-dimensional 82 model of the patient's unique anatomy [5, 6]. With image-free navigation systems, the 83 patient's anatomy is determined through measurements of anatomic landmarks and 84 joint kinematics in three dimensions [7]. For reasons of cost and convenience, image85 free navigation systems have become the most common navigation technique and will 86 be the focus of the work that we are presenting. 
88 Image-free systems utilize anatomic mapping of the joint through calculation of 89 algorithms based on specific anatomic reference points [8]. The anatomic reference

90 frames are based around the mechanical axes of the bones that make up the joint. In the

91 case of the knee (or stifle) joint, the positions of the femur and tibia are recorded in real92 time through the use of optical marker arrays that are rigidly fixed to the diaphysis of 93 the bone and tracked by a camera. The mechanical axis of the femur is determined by 94 locating the center of the femoral head, by circumduction of the femur and identification 95 of the point in space around which the femur rotates, and the center of the stifle joint, 96 through use of a digitizing stylus to identify anatomic landmarks on the distal femur and 97 proximal tibia. The tibial mechanical axis is defined proximally by the center of the stifle 98 joint (identified as above) and distally as the mid-point between the medial and lateral 99 malleoli. Once these axes have been determined, and the relationship of the marker 100 arrays to these axes has been identified, the surgeon is able to obtain real-time data 101 relating to angulation and position, allowing accurate planning of instrument placement 102 and bone preparation $[8,9]$.

104 While the utility of surgical navigation has been confirmed in laboratory and clinical 105 studies on human total knee replacement [10-12], objective data relating to the 106 relationship between component malalignment and implant failure are currently lacking 107 in canine TKR, but it is logical and reasonable to make every effort to optimize the 108 accuracy with which implants are positioned, with an expectation that this will reduce 109 the risk of implant-related complications and extend the working life of the implant. In a 110 recent cadaveric study, we demonstrated that frontal plane malalignment of 3 degrees 111 or more results in significant alterations in the loading of the medial and lateral 112 compartments of the tibial component in dogs [13]. The specific goal of the current 113 study was to build on these earlier data and to explore the feasibility and utility of using 114 computer-assisted surgical navigation in canine TKR. We hypothesized that use of 115 computer-assisted navigation would improve both the precision (reproducibility) and 116 accuracy of tibial component alignment. 


\section{MATERIALS \& METHODS}

\section{Cadaveric Tissue Specimens}

121 Retrospective data on tibial component alignment were collected from a series of 17 122 cadaveric TKR procedures performed by board-certified surgeons participating in a 123 surgical training workshop. These surgeons had experience in performing total joint 124 replacements but had not performed TKR prior to the training workshop. Tissues for a 125 prospective cadaveric study were then collected from 12 skeletally mature dogs that 126 were euthanized for reasons unrelated to this study. The pelvic limbs were 127 disarticulated at the hip and then radiographed (standard caudocranial and 128 mediolateral stifle series plus a full-length craniocaudal view of the femur) to rule out 129 the presence of pre-existing bone or joint pathology, and to allow for the measurement 130 of limb axes (see later). A $10-\mathrm{cm}$ magnification marker was included in the radiograph to 131 allow for pre-surgical templating and selection of an appropriate implant size.

133 Radiographic Determination of the Mechanical Axes of the Pelvic Limb

134 The mechanical axis of the femur was defined as a line connecting the center of the 135 femoral head proximally to the center of the intercondylar notch distally [9]. The 136 mechanical lateral distal femoral angle was defined as the angle formed between the 137 mechanical axis of the femur and the distal femoral joint surface in the frontal plane [9]. 138 The mechanical axis of the tibia was defined as a line connecting the center of the 139 intercondylar eminence proximally to a point mid-way along the distal intermediate 140 tibial ridge (Figure 1A) [14]. The mechanical medial proximal tibial angle (mMPTA) was 141 defined as the angle formed between the mechanical axis of the tibia and the proximal 142 tibial joint surface in the frontal plane (angle MPTA in Figure 1A) [10]. The sagittal axis 143 of the tibia was defined as a line joining the mid-point of the two apices of the two tibial 144 intercondylar eminences proximally and the center of a circle created by the talus 145 distally (Figure 1B) [15]. Finally, tibial slope (also referred to as the mechanical caudal 146 proximal tibial angle, mCPTA, or the tibial plateau angle) was defined as the angle 147 formed between the sagittal axis of the tibia and a line connecting the cranial and caudal 
148 margins of the articular surface of the medial tibial plateau (angle CPTA in Figure 1B) 149 [16].

Digitization and Real-time Tracking with the Surgical Navigation System

152 The procedures used for establishing surgical navigation on the canine stifle joint are

153 illustrated in Figure 2. The 12 cadaveric pelvic limbs were instrumented with retro154 reflective optical trackers rigidly attached to Ellis pins implanted into the diaphyses of 155 the femur and tibia (Figure 2A). Anatomic coordinate systems were established for the 156 femur and tibia, similar to the methods we have described previously in navigated 157 human TKR [17]. The three-dimensional positions of these marker arrays were tracked 158 in real time using a custom navigation system comprised of a Polaris Spectra camera 159 (NDI, Waterloo, Ontario, Canada) that was controlled by MATLAB (MathWorks Inc., 160 Natick, MA) and LabVIEW (National Instruments Corporation, Austin, TX) software. 161 According to the manufacturer, the measurement error associated with tracking tools 162 with this system is $0.3 \mathrm{~mm}$ root mean square (RMS). The anatomical landmarks for 163 digitization of the femur were: the center of the femoral head; the most proximal extent 164 (roof) of the intercondylar notch (Figure 2B); the medial and lateral epicondyles; and 165 the distal articular surfaces of the medial and lateral femoral condyles. Landmarks for 166 the tibia were: the medial and lateral margins of the edges of the proximal tibial articular 167 surface; the center of the intercondylar eminence; the deepest points within the medial and lateral tibial condyles; and the medial and lateral malleoli distally.

\section{Surgical Procedure - Total Knee Replacement}

171 Following digitization of the anatomic landmarks, the stifle was extended and the 172 extramedullary tibial alignment guide (ETAG) positioned against the tibia as per current 173 clinical guidelines [1]. The ETAG is a telescoping tubular instrument equipped with a 174 cutting block at its proximal end. For the surgeon-guided procedure, the ETAG was 175 positioned by eye and then secured to the proximal tibia with pins. The final alignment 176 of the cutting block relative to the mechanical axis (frontal plane) and the tibial axis 177 (sagittal plane) was then recorded with the surgical navigation system. The final slope of the tibial cut is always parallel to that of the top surface of the tibial cutting block, so the 


\section{Without Track Changes}

179 orientation of the cutting block provides a surrogate marker for final alignment of the 180 tibial component. A flat, thin-bladed tracking tool (Figure 2E) was placed on the top 181 surface of the tibial cutting block to record its orientation in the frontal and sagittal 182 planes. It was therefore not necessary to resect the tibia, making it possible to "re-use" 183 the tibia for the second procedure (navigated procedure), in which real-time output 184 from the navigation system was used to determine the optimal alignment of the 185 ETAG/tibial cutting block. At the end of the second TKR procedure, the tibia was resected and a flat, spatula-like tracker was used to measure the frontal and sagittal 187 plane alignment of the ostectomized surface of the tibia. The stifle joints were then 188 radiographed to allow measurement of tibial component alignment (see earlier description for greater detail).

Radiographic Determination of Tibial Component Alignment

192 Caudocranial and mediolateral radiographs were made following completion of the TKR 193 procedure in the specimens from the cadaveric workshop and the twelve instrumented 194 cadaveric stifles (see later). Alignment of the tibial component was measured in both the 195 frontal plane and in the sagittal plane. In the frontal plane, varus-valgus angulation of the tibial component was determined by measuring the angle formed between the mechanical axis of the tibia and a line drawn along the ostectomized surface of the tibia (Figure 3A). Sagittal plane alignment was determined by measuring the angle formed between the ostectomized surface of the tibia and the sagittal axis of the tibia (Figure 3B). As per clinical guidelines [1], the optimal orientation of the tibial component was defined as neutral varus-valgus angulation (i.e. 90 degrees relative to the mechanical axis of the tibia) and six degrees of caudal slope (i.e. 84 degrees relative to the sagittal axis of the tibia) [1].

\section{Comparison of Mechanical Axes - Radiography versus Surgical Navigation}

207 the mechanical axes of the pelvic limb, it was important to determine whether the computer-assisted tracking system was capable of accurately identifying the mechanical 


\section{Without Track Changes}

210 compared against those derived by direct measurement on the cranio-caudal 211 radiographs.

212

\section{Statistical Analysis}

214 The relationship between radiographic and navigation-assisted measurements of the

215 mechanical axes of the tibia was determined by linear regression analysis. Absolute 216 errors in cutting block alignment, relative to the gold standards of $0^{\circ}$ varus-valgus 217 angulation and $6^{\circ}$ caudal slope, were measured by the computer and data from the two

218 approaches (navigated, non-navigated) were compared by both paired t-test (to 219 compare mean values) and Levene's test (to compare variances). Intra-observer 220 variability in the measurement of tibial component alignment was determined by 221 calculating the coefficient of variation for ten repeated measurements performed by a 222 single investigator on one set of radiographs. Inter-observer variability was calculated 223 as the coefficient of variation from twelve sets of radiographs that were reviewed by 224 three independent evaluators. A significance level of $p<0.05$ was used throughout. 


\section{RESULTS}

Retrospective Analysis of Tibial Component Alignment

229 In a series of 17 cadaveric TKR procedures, the tibial component was implanted at an 230 average of $90.2^{\circ}$ (range $85^{\circ}$ to $95^{\circ}$ ) relative to the mechanical axis in the frontal plane, 231 with 2 cases (12\%) falling outside the $3^{\circ}$ error threshold (Figure $4 \mathrm{~A}$ ). In the sagittal 232 plane, the tibial component was implanted at an average of $82.4^{\circ}$ (range $78^{\circ}$ to $86^{\circ}$ ) 233 relative to the tibial axis, with 4 cases (24\%) falling more than three degrees outside the 234 optimal $6^{\circ}$ alignment that is recommended clinically [1] (Figure 4B). Inter-observer 235 reproducibility was good, with a coefficient of variation of $0.54 \%$ for alignment in the 236 frontal plane and $1.16 \%$ in the sagittal plane. Inter-observer variability was also low, 237 with a mean coefficient of variation of $1.14 \%$ (range 0 to $2.21 \%$ ) for alignment in the frontal plane and $1.97 \%$ (range 0.70 to $2.52 \%$ ) in the sagittal plane.

Use of Navigation to Determine Tibial Mechanical Axes

241 The mean $( \pm S D)$ angle formed between the tibial mechanical axis and the proximal tibial 242 joint surface was measured at $91.2^{\circ} \pm 2.2^{\circ}$ by radiography and at $91.6^{\circ} \pm 3.5^{\circ}$ by surgical 243 navigation. There was a statistically significant relationship between radiographic and navigation estimates of the tibial mechanical axis $\left(r^{2}=0.866, p<0.001\right)$ (Figure 5 ).

\section{Use of Navigation to Align the Tibial Cutting Block}

Under surgeon-guidance, the tibial cutting block was placed with a mean varus-valgus angulation of $1.24^{\circ}$ (range $-2.35^{\circ}$ to $+1.93^{\circ}$ ) in the frontal plane and a mean sagittal slope angulation of $7.08^{\circ}$ (range $+2.23^{\circ}$ to $+10.81^{\circ}$ ). With computer-assisted navigation, cutting blocks were placed with a mean varus-valgus angulation of $0.41^{\circ}$ (range $-0.71^{\circ}$ to $+0.81^{\circ}$ ) and a mean sagittal slope angulation of $6.46^{\circ}$ (range $5.13^{\circ}$ to $\left.7.59^{\circ}\right)$. When the mean values from the two techniques were compared using a paired t-test, a statistically significant improvement in alignment was identified for the frontal plane $(p=0.007)$ but not the sagittal plane $(\mathrm{p}=0.321)$. The data from the navigated procedures were less scattered than the data from the non-navigated procedures (Figure 6), and this effect 
256 was confirmed as being statistically significant by Levene's test for equality of variances

257 for both the frontal plane $(\mathrm{p}<0.001)$ and the sagittal plane $(\mathrm{p}=0.008)$.

258 


\section{Without Track Changes}

259

260

261

262

263

264

265

266

267

268

269

270

271

272

273

274

275

276

277

278

279

280

281

282

283

284

285

286

287

288

289

\section{DISCUSSION}

The results from this ex-vivo study allow us to draw two important conclusions regarding surgical accuracy in canine TKR. First, alignment errors of 3 degrees or greater were seen in a significant proportion of TKR procedures performed by surgeons performing TKR for the first time. Second, surgical navigation reduced inaccuracy and improved the precision of tibial component positioning in a cadaveric canine TKR model. Taken as a whole, these data support the potential value of integrating surgical navigation into both the training and the clinical practice of canine TKR.

Although it could be argued that the results from a surgical training workshop may not mirror those from surgeons with more experience in TKR, it is the relatively inexperienced TKR surgeon who would likely benefit most from computer-assisted surgical navigation and so we felt that it was reasonable to use this population of surgeons as the sample population for the screening portion of this study. It is highly likely that surgical accuracy increases as surgeons develop more experience with the procedure, but it is also likely that experienced surgeons will take on more challenging cases, making surgical navigation an appealing option, even if only as an objective "backup" to confirm the verify the accuracy of the procedure.

The second step of this project involved a head-to-head comparison of tibial component positioning in TKR procedures performed without surgical navigation ("surgeonguided") or with computer-assisted navigation. All of the procedures were performed by the same investigator $\square$ who had been trained in the TKR procedure by the senior author, who has performed $>145$ canine TKR procedures in vivo. Although the results from this study confirm that a traditional surgical approach (without navigation) generally results in alignment that is within the recommended guidelines, the variability of implant alignment is high. With surgeon-guided block placement, 1 of 12 dogs was aligned outside the $3^{\circ}$ threshold. With navigation, none of the 12 tibial components was malaligned in either the frontal or the sagittal plane. Blocks placed using navigation were closer to the ideal alignments in both planes. Navigation also dramatically reduced the variability in both the frontal and sagittal planes, with standard deviations in the 
290 navigated group being approximately one-third of that in the hand guided group. This

291 effect may be especially important in cases where the normal anatomy is significantly

292 deviated as a result of intra- or extra-articular pathology or deformity [18].

294 Results from human TKR have revealed that navigation systems provide more reliable 295 and reproducible mechanical axis alignment, and consistently show fewer outliers and 296 increased accuracy of alignment in navigated groups when compared to traditional 297 methods [19]. Our data from canine TKR support and parallel these findings in humans. 298 Interestingly, in addition to reducing the risk of altered joint loading, navigation has 299 been shown to improve the kinematics of total knee replacements immediately after 300 surgery [20]. It would be of interest to determine whether a similar benefit can be 301 realized in canine TKR. Improvements in joint kinematics will positively impact the 302 patient, both in terms of restoration of joint function (and a faster or more complete 303 return to activities of daily living) and by reducing the risk of implant wear and wear304 related complications such as osteolysis [21] and implant loosening [22].

306 Despite the proven advantages of computer-assisted navigation in human total joint 307 replacement, the technique has yet to be universally adopted by arthroplasty surgeons. 308 Some of the resistance relates to financial concerns over the one-time cost of equipment 309 purchase [23] and the difficulty in securing appropriate reimbursement of these costs within an increasingly constrained healthcare model. The system that we used in this study was custom built and significantly less expensive than a commercial system, with 312 a total cost of approximately $\$ 60,000$. A second source of concern relates to the risk to 313 the patient and the increase in operating room time needed for tracker placement and 314 joint registration. To date there have been minimal complications with the use of computer-assisted navigation in human TKR [24] and the available data suggest the 316 slight increase in operative time is outweighed by the improvements in surgical 317 precision that result from navigation $[25,26]$. The most significant obstacle to the routine use of navigation lies in the general attitude of experienced arthroplasty surgeons who report clinically acceptable results using non-navigated procedures. For these surgeons, the case for using navigation to improve their already excellent results is 
321 less clear. However, with ongoing concerns regarding medical malpractice, the potential

322 for documenting the accuracy of the procedure in real-time, in an electronic format that

323 can be audited and objectively assessed after the procedure, is potentially very 324 appealing.

326 In the context of potential veterinary applications, there are opportunities for using 327 surgical navigation both as a teaching tool and in clinical practice. As a training tool, 328 navigation has the potential to allow surgeons to learn the spatial relationships between 329 hand, instrument and patient within a more controlled setting. Good examples where 330 navigation could be very helpful would include acetabular reaming during total hip 331 replacement, or osteotomy alignment for the correction of angular limb deformity. Over 332 time, one would anticipate that the need for navigation will dissipate as trainees develop 333 greater confidence in their abilities, but navigation should allow surgeons to progress up 334 the learning curve with greater speed and with less risk to the patient, since the chances 335 of a clinically impactful error in implant placement will be reduced.

As a clinical tool, navigation offers potential advantages as a means of enhancing surgical accuracy and decreasing the risk of potentially problematic clinical outliers. It is widely recognized in human total joint replacement that clinical results from surgeons with a low annual total joint caseload are not as good as are those from surgeons with a high caseload $[27,28]$. With a much lower overall TKR caseload in dogs, navigation could help to ensure the quality of the procedure is consistent regardless of the interval between cases. Navigation also allows for objective documentation of implant positioning; these data could then be used in prospective studies assessing the relationship between implant alignment, joint function and implant longevity.

Given the caudal slope of the canine knee, as compared with the relatively flat tibial plateau in humans, it is likely that the canine knee is more tolerant of small errors in component angulation in the sagittal plane. This is supported by results from a recent study by Baker et al. in which the authors showed that joint kinematics and medial collateral ligament strains were similar in tibial components implanted at 8 degrees and 


\section{Without Track Changes}

352 at the recommended 6 degrees [29]. In contrast, our data on the effects of frontal plane

353 malalignment confirm that stifle loading is sensitive to errors in this plane [13]. Taken as

354 a whole, we conclude that the greatest value of navigation will likely be seen in 355 optimizing frontal plane alignment.

356

357 In conclusion, surgical navigation has the potential to reduce the number of malaligned 358 outliers by improving the accuracy and reproducibility of implant alignment. Clinical 359 studies would be required to determine whether the improvements in implant 360 alignment translate into improved clinical outcomes and implant survival in canine TKR. 361 Additionally, it will be important to determine how the accuracy of bone and joint 362 registration is influenced by the presence of osteoarthritic changes such as 363 osteophytosis and joint collapse. Further studies are now planned to address these 364 issues, as well as to evaluate the efficacy of surgical navigation in other veterinary 365 orthopedic procedures, including total hip replacement, total elbow replacement and 366 angular limb correction. 


\section{REFERENCES}

371 [1] Liska WD, Doyle ND. Canine total knee replacement: surgical technique and one-year 372 outcome. Vet Surg 2009; 38:568-582.

373 [2] Stulberg SD, Loan P, Sarin V. Computer-assisted navigation in total knee 374 replacement: results of an initial experience in thirty-five patients. J Bone Jt Surg 2002; 375 84-A Supplement 2:90-98.

376 [3] Ritter MA, Faris PM, Keating EM, et al. Postoperative alignment of total knee replacement. Its effect on survival. Clin Orthop Relat Res 1994; 299:153-156. [4] Barrack RL, Schrader T, Bertot AJ, et al. Component rotation and anterior knee pain after total knee arthroplasty. Clin Orthop Relat Res 2001; 392:46-55. [5] Stindel E, Briard JL, Merloz P, et al. Bone morphing: 3D morphological data for total knee arthroplasty. Comput Aided Surg 2002; 7:156-168. [6] Kienzle TC Jr., Stulberg SD, Peshkin M, et al. Total knee replacement. IEEE Eng Med Biol 1995; 14:301-306.

384 [7] Leitner F, Picard F, Minfelde R, et al. Computer assisted knee surgical total replacement. CVRMed-MRCAS '97: First Joint Conference Computer Vision, Virtual Reality and Robotics in Medicine and Medical Robotics and Computer-Assisted Surgery, Springer, Grenoble, France (1997), pp. 627-638

[8] Clark TC, Schmidt FH. Robot-assisted navigation versus computer-assisted navigation in primary total knee arthroplasty: efficiency and accuracy. ISRN Orthopedics 2013; 2013: 794827.

[9] Molli RG, Anderson KC, Buehler KC, et al. Computer-assisted navigation software advancements improve the accuracy of total knee arthroplasty. J Arthroplasty 2011; 26:432-438,

[10] Siston RA, Giori NJ, Goodman SB, et al. Surgical navigation for total knee arthroplasty: a perspective. J Biomechanics 2007; 40:728-735. [11] Cheng T, Zhao S, Peng $X$, et al. Does computer-assisted surgery improve postoperative leg alignment and implant positioning following total knee arthroplasty? A meta-analysis of randomized controlled trials? Knee Surg Sports Traumatol Arthrosc. 2012; 20:1307-1322. 
400 [12] Rosenberger RE, Hoser C, Quirbach S, et al. Improved accuracy of component 401 alignment with the implementation of image-free navigation in total knee arthroplasty. 402 Knee Surg Sports Traumatol Arthrosc. 2008; 16:249-257.

403 [13] Martinez MP, Bertran J, Adams A, et al. Effects of implant malpositioning on load 404 transfer following total knee replacement in dogs. 2013 Annual Conference of the 405 Veterinary Orthopedic Society, The Canyons, Park City, UT.

406 [14] Tomlinson J, Fox D, Cook JL, et al. Measurement of femoral angles in four dog 407 breeds. Vet Surg 2007; 36:593-598.

408 [15] Dismukes DI, Tomlinson JL, Fox DB, et al. Radiographic measurement of the 409 proximal and distal mechanical joint angles in the canine tibia. Vet Surg 2007; 36: 699410704.

411 [16] Dismukes DI, Tomlinson JL, Fox DB, et al. Radiographic measurement of canine 412 tibial angles in the sagittal plane. Vet Surg 2008; 37:300-305

413 [17] Siston RA, Giori, NJ, Goodman SB, et al. Intraoperative passive kinematics of 414 osteoarthritic knees before and after total knee arthroplasty. J Orthop Res 2006; 415 24:1607-1614

416 [18] Hernández-Vaquero D, Suarez-Vazquez A, Sandoval-Garcia MA, et al. Computer 417 assistance increases precision of component placement in total knee arthroplasty with 418 articular deformity. Clin Orthop Relat Res 2010; 468:1237-1241.

419 [19] Hutter EE, Granger JF, Beal MD, et al. Is there a gold standard for TKA tibial component rotational alignment? Clin Orthop Relat Res 2013; 471:1646-1653.

421 [20] Anderson KC, Buehler KC, Markel DC. Computer assisted navigation in total knee 422 arthroplasty: comparison with conventional methods. J Arthroplasty 2005; 20:132-138.

423 [21] Gallo J, Goodman SB, Konttinen YT, et al. Osteolysis around total knee arthroplasty: 424 a review of pathogenetic mechanisms. Acta Biomater 2013; 9:8046-8058. [22] Gøthesen O, Espehaug B, Havelin L, et al. Survival rates and causes of revision in cemented primary total knee replacement: a report from the Norwegian Arthroplasty Register 1994-2009. Bone Joint JSurg Br 2013; 95-B:636-642. [23] Watkins IV, R. G., Gupta, A., Watkins III, R. G. (2010). Cost-effectiveness of imageguided spine surgery. Open Orthop J 2010; 4:228-233. 


\section{Without Track Changes}

431 [24] Moskal JT, Capps SG, Mann JW, et al. Navigated versus conventional total knee 432 arthroplasty. J Knee Surg 2014; 27:235-248.

433 [25] Ulrich SD, Mont MA, Bonutti PM, et al. Scientific evidence supporting computer434 assisted surgery and minimally invasive surgery for total knee arthroplasty. Expert Rev 435 Med Devices 2007; 4:497-505.

436 [26] Dutton AQ, Yeo SJ, Yang KY, et al. Computer-assisted minimally invasive total knee 437 arthroplasty compared with standard total knee arthroplasty. A prospective, 438 randomized study. J Bone Joint Surg Am 2008; 90:2-9.

439 [27] Katz JN, Losina E, Barrett J, et al. Association between hospital and surgeon 440 procedure volume and outcomes of total hip replacement in the United States medicare 441 population. J Bone Joint Surg Am 2001; 83-A: 1622-1629.

442 [28] Losina E, Barrett J, Mahomed NN, et al. Early failures after total hip replacement: 443 effect of surgeon volume. Arthritis Rheum 2004; 50:1338-1343.

444 [29] Baker KM, Foutz TL, Johnson KJ, et al. Effects of tibial plateau angle and spacer 445 thickness applied during in vitro canine total knee replacement on three-dimensional 446 kinematics and collateral ligament strain. Am J Vet Res 2014; 75:792-799. 


\section{Without Track Changes}

447

448

449

450

451

452

453

454

455

456

457

458

459

460

461

462

463

464

465

466

467

468

469

470

471

472

473

474

475

476

477

\section{FIGURE LEGENDS}

Figure 1. Mechanical axes and joint angles of the canine tibia in the frontal (A) and sagittal (B) planes. The descriptions of the mechanical axis and the joint lines are based on those from [9] and [10] and are described more completely in the text.

Figure 2. Schematic illustration of the steps involved in surgical navigation for total knee replacement in the dog. The initial step involves implantation of rigid trackers into the femur and tibia (A). The hip is then circumducted to enable identification of the center of the femoral head, and anatomic landmarks on the distal femur (B), proximal tibia and distal tibia (C) are visually identified and marked with a tracked stylus. With the mechanical axes of the femur and tibia identified, the surgeon is able to use real-time outputs from the navigations system to ensure accurate alignment of the tibial cutting block in both frontal and sagittal planes (D). In this study, navigation was also used to document the final alignment of the tibial ostectomies in procedures that were performed without navigation assistance.

Figure 3. Radiographic determination of the accuracy of tibial preparation in the sagittal plane (A) and frontal plane (B) .

Figure 4. Variability in tibial component alignment in the frontal (A) and sagittal (B) planes. Data were collected from 17 cadaveric TKR procedures performed in surgical workshops. Red boxes identify outliers greater than 3 degrees beyond the clinically recommended alignment.

Figure 5. Relationship between tibial mechanical axis measurements derived from surgical navigation and radiography. Regression analysis $\left(\mathrm{r}^{2}=0.866, \mathrm{p}<0.001\right)$ confirmed the validity of navigation as a means of measuring limb axis alignment.

Figure 6. Effects of surgical navigation of tibial component alignment in the frontal (A) and sagittal (B) planes. Dashed line represents the \pm 3 degrees of error that were 


\section{Without Track Changes}

478 considered clinically acceptable. Data represent mean (SD) for $\mathrm{N}=12$ cadaveric samples. 479 Statistically significant differences between navigated and non-navigated groups are 480 denoted by asterisks comparisons of means by paired t-test $\left({ }^{*} p<0.05\right.$ or $\left.{ }^{* *} p<0.01\right)$ and 481 by letters for comparisons made using Levene's test for equality of variances (a 482 represents $\mathrm{p}<0.05$ and $\mathrm{b}$ represents $\mathrm{p}<0.01$ ). 